\title{
Shape and Material Selection for Optimizing Flexural Vibrations in Multilayered Resonators
}

\author{
Damiano Pasini, Member, IEEE, Member, ASME
}

\begin{abstract}
Flexural stiffness domains have been determined for optimizing the vibration frequency of a multilayered microresonator. It is demonstrated in an efficiency map that, for a given eigenvalue, the flexural properties of a two-materials system, including all possible symmetric and asymmetric multiple-layers arrangements, fall into a domain limited by two curves. These low and upper boundaries describe symmetric three-layers systems consisting of two dissimilar materials. The flexural response model adopted in this paper is based on the Euler-Bernoulli theory, and the results are obtained by extending a scheme for modeling efficiency of monolithic structures (see D. Pasini, "Shape transformers for material and shape selection of lightweight beams," $J$. Mater. Design, 2006). Shape parameters are introduced to explore the impact on the resonator vibrations of the cross-section shape, the arrangement, symmetry and number of layers, as well as the materials properties and their volume fraction. The application of the maps to a case study shows that the method can assist to exploit geometry and material potential of microstructures, especially at the concept stage of design.

[2006-0137]
\end{abstract}

Index Terms-Design methodology, frequency response, microelectromechanical systems (MEMS), microresonators, optimization methods.

\section{INTRODUCTION}

$\mathbf{M}$ ICRORESONATORS vibrate at a certain frequency when triggered by an external stimulus. Because of their sensitivity to force, temperature, and pressure changes, microresonators are exploited in an increasing number of applications for measurement, sensing, and electrostatic actuation. So far, sectors that have most benefited include telecommunication, transportation, aerospace control, information technology, and damage monitoring [1], [2]. For example, due to their high frequency sensitivity, vibrating resonators have been designed to measure the elastic properties of thin layers [3]-[6], to sense forces [7], [8], and to develop microgyroscopes [9], [10]. They have been proposed for the next generation of wireless communications and antenna applications [11]-[14], as well as exploited as micromechanical mixer-filters, oscillators, and tunable bandpass T-filters [15]-[17].

The vibration response of a resonator is a measure of its sensing capacity and its speed of actuation. It is governed by

Manuscript received July 14, 2006; revised September 11, 2006. This work was supported by the Fond Québécois de la Recherche sur la Nature et les Technologie under FQRNT NC-112230. Subject Editor S. M. Spearing.

The author is with the Department of Mechanical Engineering, Institute for Advanced Materials of McGill University, Montreal, PQ H3A 2K6, Canada (e-mail: damiano.pasini@mcgill.ca).

Digital Object Identifier 10.1109/JMEMS.2006.885997 physical attributes, such as material and geometric characteristics, and by the boundary conditions. The selection of such attributes has a large impact on resonator performance, for a faulty choice may spoil irremediably its functioning.

The structure of a microresonator may consist of one or more materials. Ceramics, metals, and polymers are often stacked strategically in multilayers. Material properties, shape, and layers geometry of a cross-section are often selected to improve certain aspect of structural performance, for they govern the relations of a number of mechanical properties, such as ductility, strength, impact toughness, and damping capacity. Structural issues, however, are not the only factors that guide design choices. In certain applications, the selection of materials and of number and configuration of layers may be imposed by other functional requirements [18]-[22]. For example, in the biomedical field, gold is often preferred as coating layer for its adsorption attributes, which are essential especially for DNA biochips. [23]-[25].

Predicting the impact that structural choices have on the vibration responses poses challenges in the design of a microresonator. The issue hinges on the designer experience and ability to foresee the advantages of opting for certain material and geometric solutions that meet given functional requirements. Such a topic has aroused significant interest among researchers. Several theoretic and computational models have been proposed, and their predictions have been validated experimentally [3]-[6], [26]-[33]. These works demonstrate that numeric methods are in better agreement with the test results. Nevertheless, theoretic models are often preferred. One of their major benefits is a drop not only of the computational time but also of the cost for long laboratory measurements.

Most of the analytical models proposed for vibration prediction have been demonstrated to be appropriate for the detailed design. They are effective in the search of the optimum value of a variable, such as the layer thickness, once a structural concept has been already decided. However, they are not tailored for the concept design. This stage requires exploring alternative geometries and material combinations before selecting the optimum candidate.

At the concept stage, design can be optimized by using criteria of excellence and selection charts. The former consists of analytical expressions that relieve the engineer from solving the entire problem. The latter plot performance curves that show how the efficiency of a solution changes with the design variables.

The underpinning rationale of a criterion of excellence is the decoupling of the variables from the constants of the problem [34]-[38]. When there is no variable interdependence, a perfor- 
mance criterion $p$ can be expressed by a product of three factors $f($.$) , collecting functional requirements F$, material properties $M$, and geometric parameters $G$, such that $p=f(F) \times$ $f(M) \times f(G)$. Such a rationale has been well exploited for material selection [38]-[44]. In this context, $F$ and $G$ are prescribed, and the index depends only on a combination of material properties such that $p=f(M)$. Various material indexes have been formulated for material selection. Ashby introduced the well-known material property charts that display one material property versus another and showed that plotting the material indexes on charts ease material selection, especially at the preliminary stage of design [38].

Because of their efficacy, material indexes and charts have been developed recently also for micromechanical design. Material indexes, formulated for the performance metrics pertinent to microdevices, permit exploiting the material potential at the microscale [42]-[47]. Such studies suggest the use of bulk material properties to be appropriate at the concept stage, as long as the microstructure size is above $1 \mu \mathrm{m}$. Although promising, these works leave mostly unexplored the issue of how to capitalize on the geometric characteristics that can boost microdevice performance.

The objective of this paper is to provide design tools for tapping into the geometry potential of a microresonator. The focus is on the impact that cross-section shape, layers arrangement, and materials have on the relations governing flexural vibration. A method that models structural performance at the mesoscale [48], [49] is here extended to microscale for a multilayered resonating beam. Based on the Euler-Bernoulli theory, the model includes the shape of a cross-section and the layers geometry as design variables. Performance measures and selection maps are developed to contrast different structural concepts and to ease the search of the optimum alternative.

Section II of this paper presents the analytical model describing the flexural vibration of a microresonator. The method for modeling the cross-sectional geometry is described in Section III, applied to the resonance frequency model in Section IV, and used in Section V to develop optimization maps. This paper concludes with a design study that shows the maps are an aid to exploiting material and geometry potential.

\section{ResonAnCE FReQUenCIES ModeL}

This section presents the flexural response model with its assumptions for a microresonating beam. Then, the model validity is discussed by resorting on numeric and experimental results drawn from peer scientific works.

\section{A. Model Assumptions}

The model described in Section II-B for predicting resonance frequency responses is based on the following assumptions [2], [45]-[47], [55].

- The resonator sizes should be greater than $1 \mu \mathrm{m}$ for the laws of continuum mechanics to be applicable.

- The materials are homogeneous and are described by their bulk properties. This hypothesis has been demonstrated to be conservative and appropriate for the early design stage.

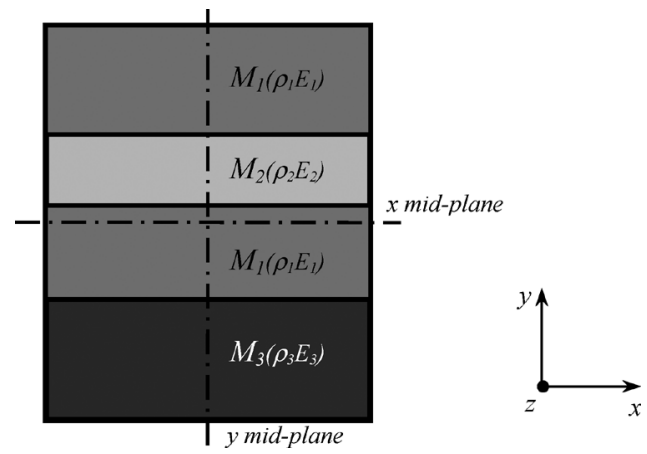

Fig. 1. System of three materials arranged in four layers.

- Euler-Bernoulli beam theory is suitable. This implies materials are isotropic; resonator cross-section is uniform along its axis; the resonator is loaded only in its plane of symmetry; deformation remains small; plane sections do not deform, i.e., transverse shear deformations are neglected; and the microstructure is long and slender with aspect ratio from $1: 10$ to $1: 20$.

\section{B. Flexural Response Model}

In this section, the resonance frequency model is presented first for a monolithic material and then for a layered system.

Consider a single material uniform beam of length $l$ in flexural vibration. The cross-section area and second moment of area of the beam are, respectively, $A$ and $I$, and the density and Young's modulus are $\rho$ and $E$. An excitation force per unit length $f(z, t)$ acts on the beam, in transverse direction, at location $z$, and it induces a deflection $v(z, t)$. The equation governing the forced transverse vibration of the Euler-Bernoulli beam [1] is

$$
\rho A \frac{\partial^{2} v}{\partial t^{2}}+\frac{\partial^{2}}{\partial z^{2}} E I \frac{\partial^{2} v}{\partial z^{2}}-f(z, t)=0 .
$$

In free vibration, $f(z, t)=0$ and the resonance frequencies of the monolithic beam can be written by solving (1) as

$$
f=\frac{\left(\Gamma_{n} l\right)^{2}}{2 \pi l^{2}}\left(\frac{E I}{\rho A}\right)^{0.5}
$$

where $\Gamma_{n} 1$ is a length parameter determined by the order mode of vibration and by the boundary conditions. For example, $\Gamma_{l} l=$ 1.875 provides the lowest resonance frequency of a clamped cantilever.

Consider now a multilayered system consisting of different materials. Fig. 1 shows an example. The cross-section is rectangular, with three materials arranged in four layers. Equation (2) is still suitable to predict flexural vibration of a multilayered beam as long as $E I$ and $\rho A$ are replaced by transformed properties $E_{T} I$ and $\rho_{T} A$ that take into account geometry and material of layers. Thus, for a beam consisting of $k$ layers with 
$y$ mid-plane symmetry, Young's modulus $E_{i}$, and density $\rho_{i}$, the transformed flexural stiffness and unit mass are, respectively

$$
\left\{\begin{array}{l}
E_{T} I=\sum_{i=1}^{k} E_{i} I_{i}=\sum_{i=1}^{k} E_{i} \int_{A_{i}} y_{i}^{2} d A \\
\rho_{T} A=\sum_{i=1}^{k} \rho_{i} A_{i}
\end{array}\right.
$$

where $y_{i}$ is the distance from the beam $x$-mid-plane to the centroid of each layer $i$, with area and second moment of area, respectively, $A_{i}$ and $I_{i}$.

To obtain the flexural response of a multilayered system, (3) is substituted into (2) such that

$$
f=\frac{\left(\Gamma_{n} l\right)^{2}}{2 \pi l^{2}} \frac{E_{T} I}{\rho_{T} A}=\frac{\left(\Gamma_{n} l\right)^{2}}{2 \pi l^{2}}\left(\sum_{i=1}^{k} \frac{E_{i}}{\rho_{i}} \frac{\int_{A_{i}} y_{i}^{2} d A}{A_{i}}\right)^{0.5} .
$$

The next section discusses the validity of this model for microresonator design.

\section{Numeric and Experimental Data for Model Validation}

Equation (4) can predict the resonance frequency of a layered system that satisfies the assumptions listed in Section II-A. Examining the validity of such a model is crucial to the design of a microresonator, especially for high-precision applications. For frequency prediction, similar methods based on the Euler-Bernoulli theory have been proposed, and their accuracy has been verified both numerically and experimentally [4]-[7], [26]-[33]. This section discusses these recent studies for single and multilayered resonators to set the validity boundaries of the results given in this paper. The works discussed here focus on monolithic as well as multilayered beams with constant and variable laminate thicknesses.

The Euler-Bernoulli and the Timoshenko theories have been used to model the fundamental frequency of free-free and clamped-clamped resonators [26]. Uniform polysilicon microbeams with different dimensions have been designed for resonance frequencies of 30,50,70, and $90 \mathrm{MHz}$. The goal of this work was to study the effect of the structural supports on the quality factor for very high resonance frequency (VHF) beams. The results obtained from both of the models have been contrasted with the measures from computational and experimental analysis. Their data show that the Timoshenko model is more accurate, above all for upper VHF flexural mode. This is manifest especially when the resonator has an aspect ratio less than $1: 10$ and, thus, fails to meet Euler-Bernoulli assumptions. In such a case, Timoshenko theory is essential to take into account the plate effects, although shell theory can still be accurate if the plate thickness is thin [27], [28]. Simple beam predictions, however, may deviate by a maximum of $3 \%$ from the test measures, as long as the hypotheses are satisfied [26].

With respect to its assumptions, the Euler-Bernoulli beam equation has been shown suitable also for the design of multimaterial resonators [29]. A multilayered zirconate titanate piezoelectric uniform beam has been designed for a natural frequency range of $448 \mathrm{kHz}-1.1 \mathrm{MHz}$. In this paper, the beam length and the corresponding anchor length are varied discretely in the range 110-174 and 537-1408 $\mu \mathrm{m}$, respectively; on the other hand, layer thicknesses are kept constant. The theoretical predictions are shown to be in good agreement with finite-element analysis but to be lower than the test measures. The discrepancy, though, has been attributed to the uncertainty of the bulk material properties and, partially, to the imperfect anchor conditions.

By means of the Euler-Bernoulli model, the first five order flexural modes have been estimated for a multilayered beam [30]-[32]. The studies that investigate the effect of a gold film on the sensitivity of silicon dioxide in layered cantilevers have been experimentally validated. Whereas in Ferguson's work the layer thicknesses are kept constant [29], in Sandeberg's [30]-[32], the gold layer coating $\mathrm{SiO}_{2}$ is changed discretely among the values $0,100,200$, and $400 \mathrm{~nm}$, and the cross-section sizes are constrained. The analytical predictions diverge from the test measures within the range $0.71-1.5 \%$. The deviation of the results is attributed to the inaccuracy in determining layer thicknesses and material properties of the samples. The good agreement found in this paper between experimental results, finite-element model, and analytical model is evidence of the validity of simple beam theory to design a multimaterial resonating system with different layer thicknesses.

Similar conclusions can be drawn from a study on the dependence of resonance frequency from pressure and temperature for bimaterial resonators in atmospheres of helium and dry nitrogen [33]. As in Sandeberg's works [30]-[32], four different thicknesses, i.e., 0, 60, 160, and $260 \mathrm{~nm}$, have been chosen for the gold film coating silicon nitrade. Based on continuum mechanics, the results show that at a pressure of $10^{-2} \mathrm{~Pa}$, the maximum frequency variation between experiment measures and model values is $1.8 \%$.

The above studies and other experimental works [5]-[8] prove that as long as the assumptions are respected, the accuracy of Euler-Bernoulli prediction diverges no more than $3 \%$ from experiment measures. The deviation can be usually attributed to design uncertainties, such as material properties, boundaries conditions, thickness measures, and anchor dissipation. Such an estimate can be considered adequate for the concept design of microstructures.

In Section IV, Euler-Bernoulli theory is used to model the vibrations of alternative shape layered geometry. The scheme to include these variables is described in the following section.

\section{The Method of Shape Transformers}

This section presents a method that characterizes the geometry of a cross-section. So far, the approach has been applied to characterize the transversal geometry of a monolithic structure at the mesoscale [48], [49]. In this paper, the method is extended to a multimaterials microsystem.

\section{A. Cross-Section Constituents}

Size and shape govern the geometry of a cross-section. The scheme adopted here proposes to decouple the size from the shape of a cross-section and assigns to them two distinct connotations. The former is the envelope $D$, a rectangle with the dimensions of the cross-section; the latter is the shape $S$, the 


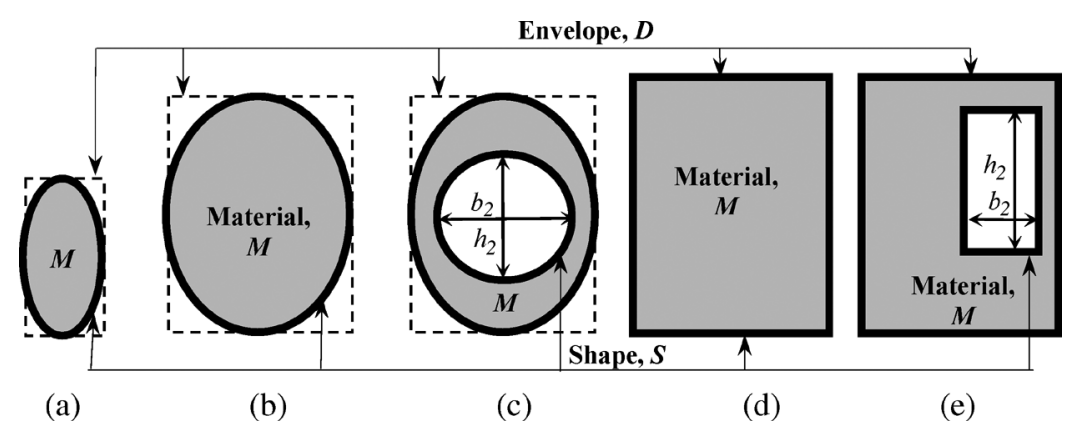

Fig. 2. Material, shape, and envelope of five cross-sections.

figure enclosed in $D$. Envelope and shape are illustrated for five different cross-sections in Fig. 2. Fig. 2(a) and (b) shows cross-sections with the same shape but different envelope. The cross-sections depicted in Fig. 2(b) and (c) have the same $D$ as those shown in Fig. 2(d) and (e). Fig. 2(c) illustrates the particular case of the solid rectangle, where the shape fills completely the envelope, i.e., $D \equiv S$.

The reasons for decoupling $S$ from $D$ are manifold. First, $D$ and $S$ are variables governing two different aspects of cross-section geometry. $D$ is in charge of ruling size changes. $S$ specifies shape properties that are invariant to cross-section scaling. Second, such an approach helps identify the role that each variable plays in an equation of continuum mechanics. Third, it eases the development of visual maps for material and shape selection, regardless of changes of a structure size. Last, the approach provides insight into the selection of the parameters that best improve performance.

The following section introduces the scalar operators describing envelope scaling and cross-section shaping.

\section{B. Envelope Multiplicators}

Size changes between cross-sections, e.g., Fig. 1(a) and (b), can be determined by means of two envelope multiplicators $u$ and $v$. If $B_{o}$ and $H_{o}$ are the width and height of a reference rectangle, the scaling relations of a generic envelope $(B, H)$ relative to the reference are established by

$$
\left\{\begin{array}{l}
u=\frac{B}{B_{o}}=\frac{b_{2}}{B} \\
v=\frac{H}{H_{o}}=\frac{h_{2}}{H}
\end{array}\right.
$$

where $b_{2}$ and $h_{2}$ are the internal dimensions of hollow crosssections [Figs. 2(c) and (e)].

\section{Shape Transformers}

For a given geometric quantity, such as the area, we define a shape property that is invariant to size changes of the cross-section. Such a property is described by a dimensionless parameter, a shape transformer, that measures certain aspects of the cross-section geometry. It is obtained by normalizing the geometric quantity $G$ by the same geometric quantity $G_{D}$ of the envelope such that

$$
\psi_{G}=\frac{G}{G_{D}}
$$

In (4) describing vibration frequencies, the material properties are density and Young's modulus, whereas the geometric quantities $G$ are area and second moment of area. Therefore, the shape transformers relevant to (4) can be obtained from (6) for $A$ and $I$ such that

$$
\left\{\begin{array}{l}
\psi_{A}=\frac{A}{A_{D}}=\frac{\int_{A} d A}{B H} \\
\psi_{I}=\frac{I}{I_{D}}=12 \frac{\int_{A} y^{2} d A}{B H^{3}}
\end{array} .\right.
$$

The shape and material properties of a resonating beam are, respectively, $\left(\psi_{A}, \psi_{I}\right)$ and $(\rho, E)$. The former are analogues to the latter because, generally, they are both invariant to scaling.

Table I reports the shape attributes of alternative concepts, with at least one axis of symmetry averting asymmetric bending. It is noted that asymmetric bending arises in cross-sections that do not possess any axes of symmetry; thus such beams are not considered here. Furthermore, for y-midplane symmetric crosssections, $\mathrm{x}$-mid-plane asymmetry is modeled with the term PA in the expressions of the shape transformers given in Table I. $\mathrm{PA}=0$ if the layers are symmetrically arranged about the $\mathrm{x}$-envelope midplane. As can been seen, $\psi_{A}$ and $\psi_{I}$ characterize the area and second moment of area of a shape concept, regardless of its size. Each concept has a specific range, with values that vary with respect to the area filling the envelope. When the material saturates completely the shape, we have a solid shape, and $\left(\psi_{A}, \psi_{I}\right)$ assume the range upper bounds; whereas for hollow shapes, $\left(\psi_{A}, \psi_{I}\right)$ may decrease up to zero, which corresponds to an empty shape. For example, $\psi_{A}=\psi_{I}=1$ are the properties of a solid rectangle [see Fig. 2(d)], the stiffest among constrained cross-sectional shapes. For hollow rectangles, $\left(0<\psi_{A}<1,0<\psi<l\right)$. For other solid shape concepts, e.g., those given in Table I, $\psi_{A}$ and $\psi_{I}$ are constants less than one. A solid ellipse, for instance, has always properties $\left(\psi_{A}=\pi / 4, \psi_{I}=3 \pi / 16\right)$ regardless of any envelope scaling. For their respective hollow ellipses, the shape transformers vary, but always within the ranges $\left(0<\psi_{A}<\pi / 4\right.$, $\left.0<\psi_{I}<3 \pi / 16\right)$

\section{A Shape Classification}

Shape transformers have been used to define families and classes of shapes, in a way similar to material classification [49]. Several families can be defined. Three of them have been included in Table I. The description of such a taxonomy follows. 


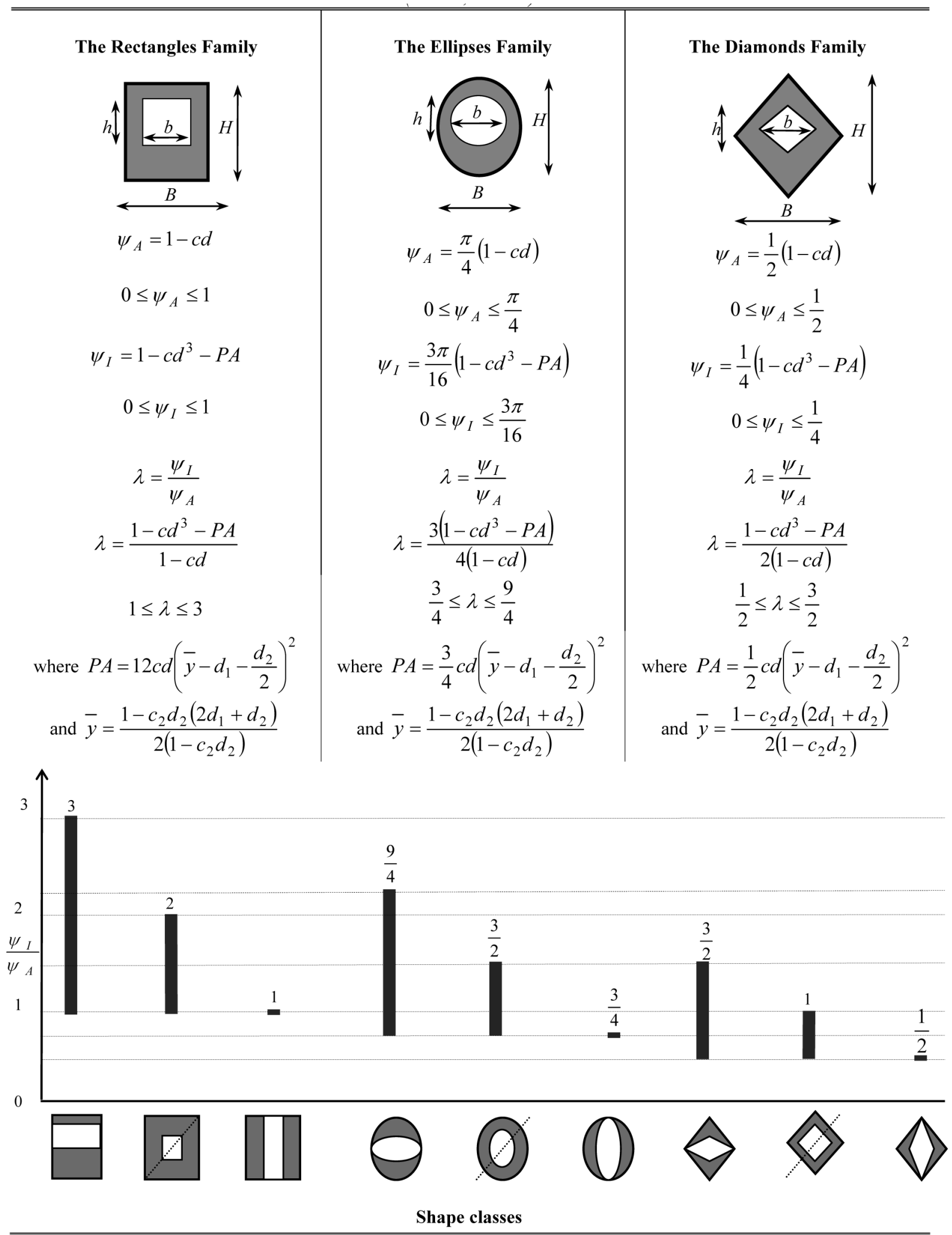

Families: A family describes a concept for the shape of a cross-section. In the scheme adopted here, families are identified by the name of a common solid shape, e.g., the family of Rectangles and the family of Ellipses (Table I). Members of a family, however, do not need to be solid.
Hollow shapes fall into a family, as long as both the shapes of the internal and the external contours are the same, regardless of the position of the hole within the cross-section. For example, the cross-section shown in Fig. 2(c) has an elliptical hole and thus belongs to the Ellipses family. 
Fig. 2(e), on the other hand, illustrates a member of the Rectangles family, where the shape of the removed internal material is rectangular and arbitrarily located within the Envelope.

Classes: The filter chosen to sort the members of a family into classes is the scaling relation between the size of the internal layer and the Envelope of the cross-section. Hence, a mode for scaling layers characterizes a class of a family, as shown for the families of Rectangles, Ellipses, and Diamonds in Table I. For each family, classes are identified with respect to three scaling modes applied to the internal layers: vertical $(c=1)$, proportional $(c=d)$, and horizontal $(d=1)$.

Similar to materials, shapes of a family exhibit properties that fall into a particular range, as shown in Table I. Using such ranges may help in the early stage of optimization. For example, combining attributes, such as $E / \rho$, permits one to select the lightest material for a limited stiffness beam constrained in size. In an analog way, the shape properties ratio $\psi_{I} / \psi_{A}=\lambda$ is the envelope efficiency parameter, which measures the efficiency of material distribution within the envelope. Specific $\lambda$ thresholds characterize a class and, thus, permit efficiency comparison between shape concepts, as shown at the bottom of Table I. The higher $\lambda$ is, the stiffer and lighter is the shape. These ranges, however, are theoretical because there are manufacturing constraints, shear failure requirements, and local instability that limit the shaping of a monolithic material into a thin wall.

\section{E. The Fundamental Rationale}

The underlying principle of the scheme of the shape transformers is the decoupling of $S$ from $D$. Consider an equation of mechanics, such as the stiffness of an element. This is often expressed by a product $F \times M \times G$, where $F$ describes the problem specifications, $M$ the material properties, and $G$ an aspect of the geometry. Applying (1) enables to reformulate the equation as a product of four factors, i.e., $F \times M \times \psi_{G} \times G_{D}$. For example, $l \times \rho \times A$ is the mass of a uniform beam of length $l$, and it can be reexpressed simply as $l \times \rho \times \psi_{A} \times A_{D}$

Such an approach has been demonstrated to ease material and shape selection for lightweight design [48], [49]. Unlike previous methods, it allows the general formulation of selection indices for any cross-section scaling. In addition, the method exploits the duality between material and shape properties and permits the development of properties maps. The latter display the performance of different shape and material concepts in a snappy form. So far, the rationale has been applied to optimize the lightweight potential of a single structured material. In this paper, the model is extended to a multimaterials microsystem.

\section{Flexural Response Model With Shape TRANSFORMERS}

In this section, the shape transformers are used to characterize the geometry of a multilayered cross-section and to reformulate the frequency response Euler-Bernoulli model.
Consider a system of generic cross-section shape, consisting of multiple deposited thin films. The prescribed parameters are length, envelope size, boundary conditions, and eigenmode of the resonator. The geometric variables are the layers pattern and the shape of the cross-section. Both of them are now modeled separately.

\section{A. Layers Pattern}

To characterize the layers arrangement, we start assuming the shape is prescribed. We chose a rectangular cross-section $\left(\psi_{A}=\right.$ $1, \psi_{I}=1$ ) with flexural rigidity $E_{T} I=E_{T} I_{D}$ and unit mass $\rho_{T} A=\rho_{T} A_{D}$. Dividing (3) by $I_{D}$ and $A_{D}$ and substituting (7), we obtain the transformed properties that model a rectangular layered system

$$
\begin{aligned}
& E_{T}=\sum_{i=1}^{k} E_{i} \psi_{I i} \\
& \rho_{T}=\sum_{i=1}^{k} \rho_{i} \psi_{A i} .
\end{aligned}
$$
be

Replacing (8) and (9) in (4) yields the vibration frequency to (1)

$$
f=\frac{H\left(\Gamma_{n} l\right)^{2}}{2 \pi l^{2}}\left(\frac{E_{T}}{\rho_{T}}\right)^{0.5}=\frac{H\left(\Gamma_{n} l\right)^{2}}{2 \pi l^{2}}\left(\sum_{i=1}^{k} \frac{E_{i} \lambda_{i}}{\rho_{i}}\right)^{0.5}
$$

where the transformed properties $\left(\rho_{T}, E_{T}\right)$ take into account material $\left(\rho_{i}, E_{i}\right)$ and geometry $\left(\psi_{A i}, \psi_{I i}\right)$ of each layer $i$. Thus, for a constrained resonator of the Rectangles family with given eigenmode, boundary conditions, and length, the first quotient in (10) is constant and the resonance frequency is governed by $E_{T} / \rho_{T}$.

\section{B. Cross-Section Shape}

If, in addition to layer geometry, the shape of a cross-section is also a variable, then the generic shape is described by properties $\left(\psi_{A} \neq 1, \psi_{I} \neq 1\right)$. Hence, applying the shape transformers to a rectangular system consisting of $k$ layers yields (8) and (9) to be

$$
\begin{aligned}
& E_{T}=\underbrace{\psi_{I}}_{S} \underbrace{\sum_{i=1}^{k} E_{i} \psi_{I i}}_{L} \\
& \rho_{T}=\underbrace{\psi_{A}}_{S} \underbrace{\sum_{i=1}^{k} \rho_{i} \psi_{A i}}_{L}
\end{aligned}
$$

where $S$ refers to the shape of the cross-section and the group $L$ describes the geometry and material of each layer. 


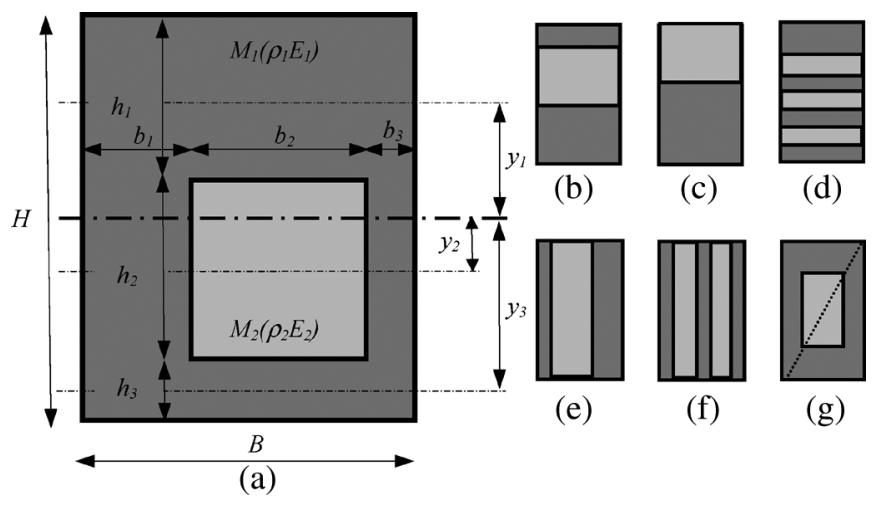

Fig. 3. (a) Full coated bimaterial; such a cross-section can evolve in the alternative multilayered geometries shown in $(\mathrm{b})-(\mathrm{g})$.

Finally, substituting (11) and (12) in (4) gives the Euler-Bernoulli model function of the shape transformers

$$
f=\frac{H\left(\Gamma_{n} l\right)^{2}}{2 \pi l^{2}}\left(\frac{\lambda E_{T}}{\rho_{T}}\right)^{0.5}=\frac{H\left(\Gamma_{n} l\right)^{2}}{2 \pi l^{2}}\left(\lambda \sum_{i=1}^{k} \frac{E_{i} \lambda_{i}}{\rho_{i}}\right)^{0.5} .
$$

For a shape multilayered resonator with given length, envelope, boundary conditions, and eigenvalue, the resonance frequency is governed by the index $\lambda E_{T} / \rho_{T} . \lambda$ and $E_{T} / \rho_{T}$ can characterize several shapes and layers geometries. Table I provides a few of the former, and Table II includes expressions $\left(\rho_{T}\right.$, $E_{T}$ ) for the latter. These are given, as an example, for a bimaterial three-layers system of the Rectangles family.

\section{Bimaterial Rectangular System EVOlVing in MULTIPLE LAYERS}

Often, microresonators are fabricated as a layered composite of laminated thin films. These can be arranged in alternative layouts that impact the flexural responses of the resonator. The focus of this section is on alternative layer geometries. We examine a two-materials system and explore how such a system can evolve into several multilayered configurations. To do so, we benefit from the shape classification described in Section III.

Consider a generic bimaterial system of the Rectangles family, such as that illustrated in Fig. 3(a). Its core is fully coated, and the coating layer has nonuniform thickness. Such a bimaterial can evolve in a large number of layouts. A few of these have been depicted in Fig. 3(b)-(g). The evolved multilayered geometries can be classified into classes. As explained, the common feature of class members is the relative scaling of layers. Within a class, however, members may differ for number and asymmetry of the layers, as well as for the relative concentration of materials. For the latter, there are two limit cases. The first one is when the percentage of material 2 is increased to 100, and the system degenerates in a monolithic material. The other one is when material 1 is the only filler, and the resonator is uncoated with $\left(\rho_{T}, E_{T}\right)$ that equals $\left(\rho_{1}, E_{1}\right)$.

For microresonators, neither any shape nor every evolved layout is achievable. Some geometry should be considered as theoretical cases, because current fabrication techniques will not be able to machine them. This issue and that of residual stresses between thin deposited films plays a major role in micromechanical design. These factors impact many mechanical properties, including the flexural stiffness, reduce the range of feasible geometries, and limit the precision of the microstructure. However, if such constraints are discarded at a preliminary design stage, then we could start to sort alternatives into classes and assess the efficiency of the concepts. For instance, the common sandwich consisting of three or more layers [Fig. 3(b)-(d)] falls into the heightwise scaled layers class. An alternative may be the full encapsulated cross-section Fig. 3(g), which belongs to another class. This geometry has been demonstrated advantageous to reduce the resonator sensitivity to temperature [22]. Another viable solution for certain applications is a layout with the core coated sideways, a concept of the widthwise scaled layers class [Fig. 3(e) and (f)].

Equation (10) can predict the flexural response for all the layout alternatives described above and (13) for those with different cross-section shape. As an example, Table II includes a few layouts with three layers. Besides $\left(\rho_{T}, E_{T}\right)$, ranges of $E_{T} / \rho_{T}$ are provided for each class. As can be observed, the thresholds resemble those given in Table I, and, similarly, they help contrast the potential of bimaterial alternatives. Displayed in an efficiency map, the transformed properties show that the resonance frequency for all the possible bimaterial systems evolving in multilayers falls into a specific domain. This is shown in the next section.

\section{PROPERTIES MAPS}

Material properties charts have been introduced by Ashby to assist material selection [38]. In a compact form, they display the relation between the properties describing material performance for a given application. On a material chart, materials of a class cluster together in regions limited by a range of material properties. The selection of the best material is guided by lines of isoperformance that are superimposed for different design conditions.

Similar to the Ashby's charts, shape property maps have been developed to visualize the shape classification described in Section III and to guide shape selection [48], [49]. A shape efficiency map plots the transformers that govern the shape performance of families and classes. Members of a family group together in a domain limited by a specific properties range (Table I), and shape selection is controlled by isoperformance lines.

Combining material charts with shape efficiency maps illustrates the impact of shaping a material into different families and classes [49]. The rationale described in Section III-E permits to contrast, in a glance, candidate performance and to choose the best combination of shape and material.

The following section presents the use of efficiency charts for optimizing the frequency responses of a bimaterial system.

\section{A. Resonance Domains of the Rectangles Classes}

$\left(\rho_{T}, E_{T}\right)$ in Table II can describe the transformed properties of the Rectangles classes consisting of two materials arranged in three layers. $E_{T} / \rho_{T}$ is the index governing the vibra- 
TABLE II

Property CHARACTERIZATION FOR a ReCTANGUlar Two-Materials SYSTEM $\left(c_{1}=b_{1} / B\right.$ and $d_{1}=h_{1} / H ; c_{2}=b_{2} / B$ and $\left.d_{2}=h_{2} / H ; n=E_{2} / E_{1} ; m=\rho_{2} / \rho_{1}\right)$

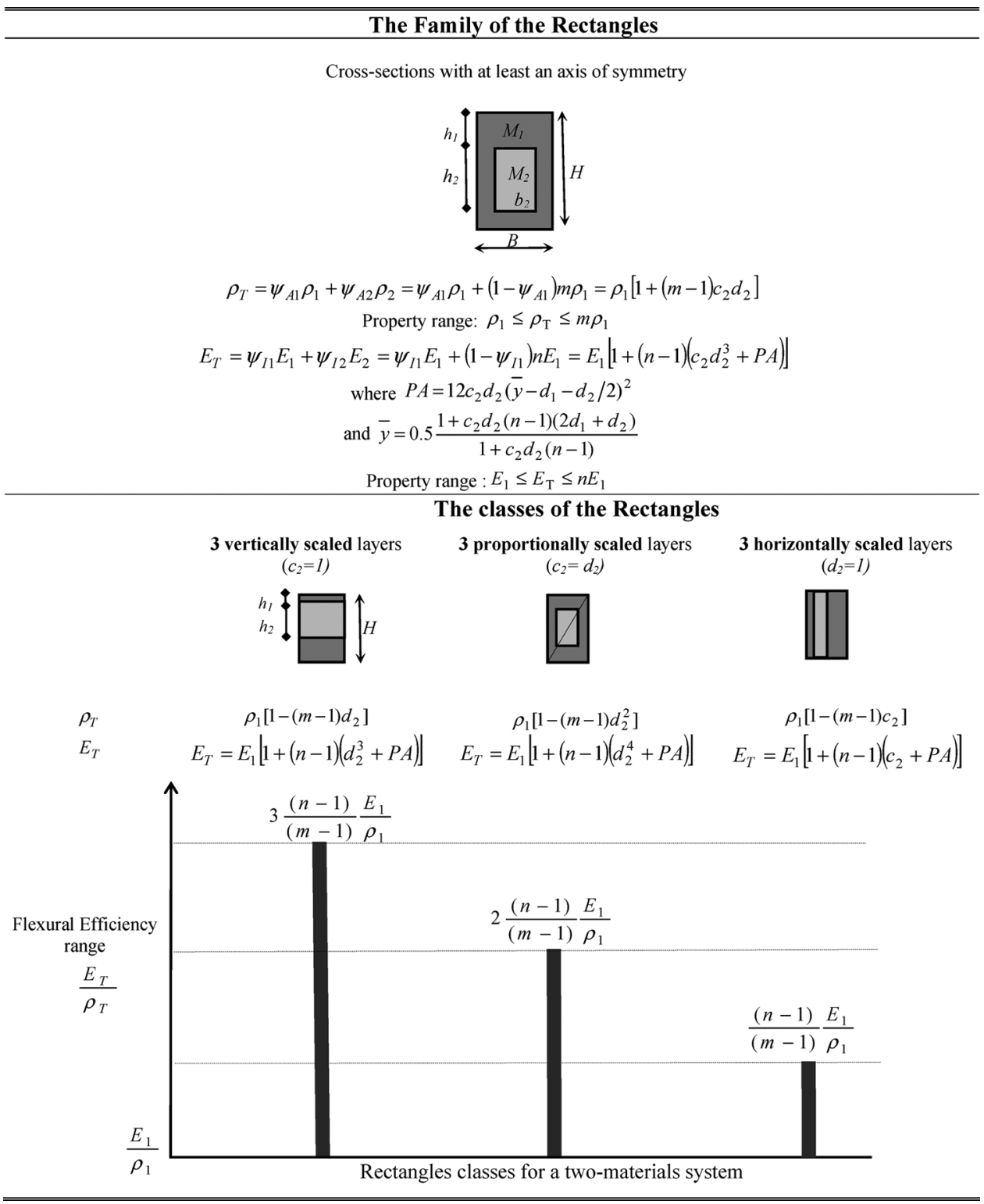

tion response for a given eigenmode, boundary conditions, beam length, and size. Hence, plotting $E_{T}$ versus $\rho_{T}$ on normalized axes, permits one to visualize $E_{T} / \rho_{T}$ as the slope of the line passing through a point and the origin. The greater the slope, the higher the vibration frequency. This is shown in Figs. 4-6.

Consider, as an example, a bimaterial cross-section with density ratio $m=\rho_{1} / \rho_{2}=2\left(\rho_{1}>\rho_{2}\right)$ and Young's modulus ratio $n=E_{2} / E_{1}=3\left(E_{2}>E_{1}\right)$. Using $\left(\rho_{T}, E_{T}\right)$ given in Table II, the plot in Fig. 4 shows that the resonance frequency efficiency is bounded by two limiting monotonic curves. The first curve $v 1$ is the upper bound. It describes symmetric three-layers beams with the stiffer material far from the neutral axis. As we said, this sandwich concept is the most common and efficient solution for a layered system. The other curve is the lower bound $v 2$, and it represents symmetric shapes with low frequency vibration responses. Here, the stiffer material fills the cross-section core. It is noticed that the relative ratios of the material properties $n$ and $m$ determines the orientation of the domain but does not impact the domain boundaries. For example, if $\rho_{1}<\rho_{2}$ and $E_{1}<E_{2}$, then curves $v 1$ and $v 2$ will be monotonic increasing functions, as opposed to those illustrated in Fig. 4 that have negative derivative. Bimaterial systems with different $n$ and $m$ will be examined in the case study of Section VII.

The maximum and minimum values of vibration performance that a concept can achieve are obtained by differentiating the function describing the curves. This involves that for $\rho_{T} \rightarrow \rho_{1}$, the gradient of curve $v 1$ in Fig. 4 reaches $E_{T} / \rho_{T}=3(n-$ $1) /(m-1) * E_{1} / \rho_{1}$, whereas the gradient of curve $v 2$ is zero, and thus, $E_{T} / \rho_{T}=E_{1} / \rho_{1}$. Such limits are listed in Table II and enable a quick relative ranking of layout concepts, based only on material properties.

In Fig. 4 , curves $p 1$ and $p 2$ represent the boundaries of the frequency domain for the proportionally scaled layers class. The former represents fully encapsulated symmetric cross-sections 


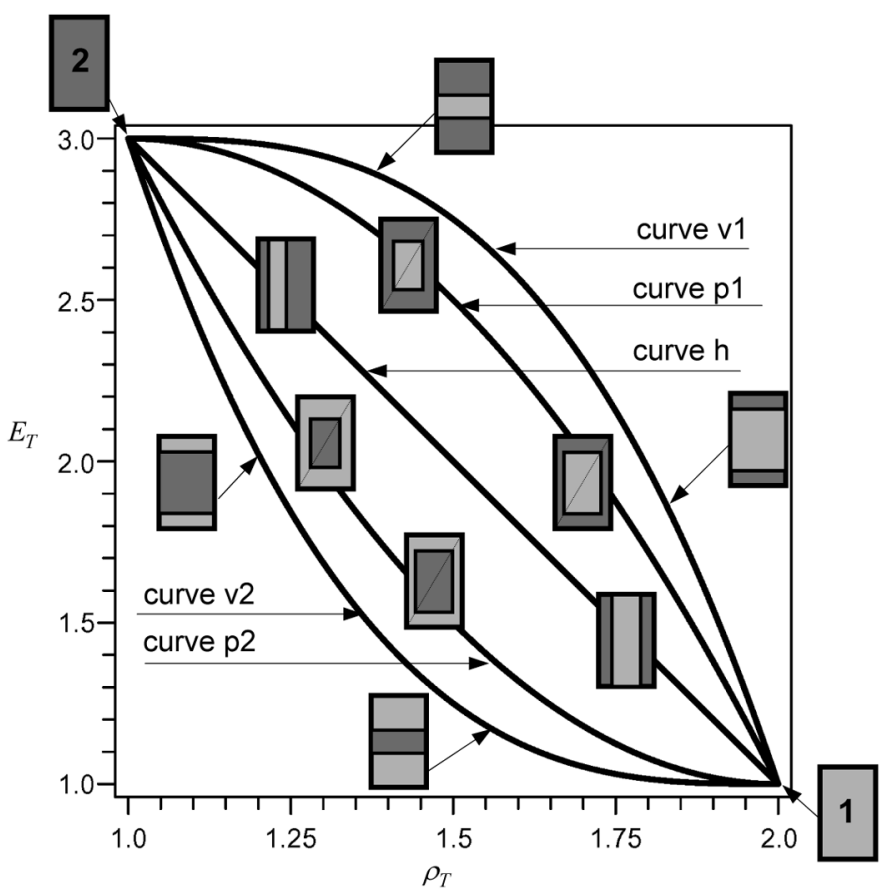

Fig. 4. Frequency response domain for the Rectangle classes of a bimaterial rectangular system. All the possible configurations of a bimaterial system are enclosed within curves v1 and $\mathrm{v} 2$ describing three symmetric layers sandwiches.

with a softer material core. For the same volume fraction, the latter characterizes stiffer material core systems. The upper efficiency bound is the maximum curve gradient, which for this class is lower than the previous one, as shown in Table II. However, as mentioned previously, a full coated resonator is preferred if the reduction of the native temperature coefficient of frequency is a design priority [22].

The third class describes widthwise scaled layers. In contrast to the others, this domain is not a region. It shrinks to a straight segment plotted as curve $h$ in Fig. 4 . The slope is $(n-1) /(m-1) * E_{1} / \rho_{1}$ for any volume fraction, as well as any number and position of layers. These factors do not impact vibrations, whereas they have a large effect on the flexural responses of different layout geometries. This is examined in the following sections.

\section{B. Impact of Multiple Layers in Symmetric Systems}

Section $\mathrm{V}$ discusses ways for a bimaterial system to evolve in multiple layers. This section explores the effect of multilayering a bimaterial system that exhibits symmetry with respect to, at least, one envelope mid-plane. The focus is on the number of layers and its impact on the flexural response. It is noted that symmetry about the envelope $x$-mid-plane yields the term PA in the expressions of the shape transformers (Tables I and II) to be zero.

To evaluate the effect of multiple symmetric layers, we restrain the volume fraction to a certain value and use (11) and (12) to obtain $\left(\rho_{T}, E_{T}\right)$. By doing so, we demonstrate that within the family domain, there exist all the possible vertically scaled symmetric layers, evolved with two, three, five, or even multiple layers. This is shown in Fig. 5 by the vertical segment $\mathrm{AB}$ plotted, as an example, for $\rho_{1} \psi_{A 1}=0.5$. This reveals that

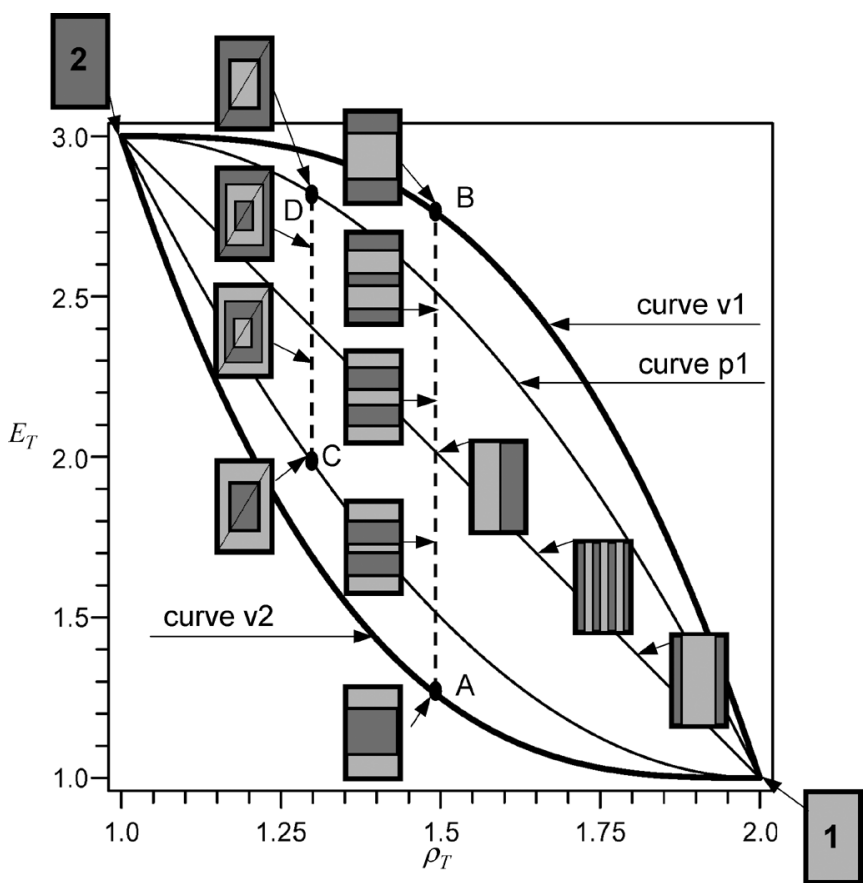

Fig. 5. Flexural responses of symmetric multilayered systems. For a given volume fraction, increasing the number of symmetric layers has an impact on the flexural vibration of fully coated cross-sections and heightwise scaled layers systems. On the other hand, no influence can be observed when layers are scaled along the width.

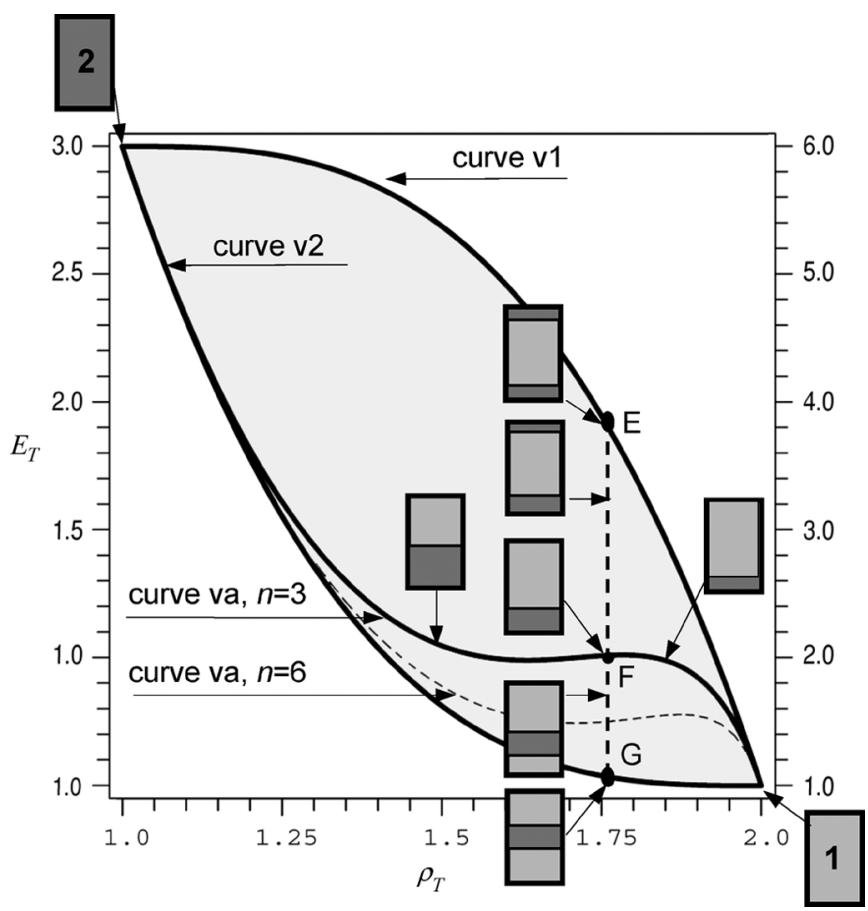

Fig. 6. Curves va show the impact of asymmetry into the flexural response of a bimaterial system. $\left(\rho_{1}=2 \rho_{2} ; E_{2}=3 E_{1}\right)$ for the ordinates on the left; $\left(\rho_{1}=2 \rho_{2} ; E_{2}=6 E_{1}\right)$ for the ordinates on the right. Increasing the Young Modulus ratio $n=E_{2} / E_{1}$ causes the flexural response curve va to approach curve v2, i.e., the resonance response of a three-symmetric-layers sandwich.

the flexural modulus of such layouts lies between, but cannot reach, $\mathrm{A}$ and B. The maximum and minimum $E_{T} / \rho_{T}$ are obtained only if the system evolves in three symmetric layers.

Applying a similar analysis to the class of proportionally scaled layers gives analogous results. Fig. 5 shows that for 
$\rho_{1} \psi_{A 1}=0.3$, the evolved layouts can have multiple nested layers, but all of them fall between $\mathrm{C}$ and $\mathrm{D}$. These points belong to the class boundaries and describe symmetric layout with only one coating layer.

Whereas the dependence of $E_{T}$ from $\rho_{T}$ is cubic for sandwich layouts, and is quadratic for fully coated concepts, $E_{T}=f\left(\rho_{T}\right)$ is linear for widthwise scaled layers. This is represented by curve $h$ in Fig. 5. Plotting (11) and (12) for systems evolving with two, three, five, or more films confirms that the number of layers does not control flexural response.

\section{Impact of $x$-Mid-Plane Asymmetry Into Multiple Layers Systems}

Once the influence of the number of layers is examined, we explore graphically the impact of asymmetric layers for a two-, three-, and multilayer systems. Unlike beams fully symmetric about both $\mathrm{x}$ - and $\mathrm{y}$-envelope mid-planes, cross-sections with $\mathrm{X}$-mid-plane asymmetry have the term $\mathrm{PA} \neq 0$ in the expressions of the shape transformers given in Table I.

For a two-layered system, $E_{T} / \rho_{T}$ depends on the Young's modulus ratio. This is shown by curve $v a$, which is plotted in Fig. 6 for $n=3$ (continuous line referring to the left $y$-axis) and $n=6$ (dashed curve referring to the right $y$-axis). From the map, it can be observed that for a two-asymmetric-layers beam, an increase of the Young's modulus ratio $n$ lowers curve $v a$ towards the limiting curve $v 2$.

For a three-layer system, curve $v a$ splits the Rectangles domain into two regions. Above there are sandwiches with softer material core, and below there are layouts with stiffer coated layer. This observation can be obtained by restraining the volume fraction to a certain value and using (11) and (12) to characterize an asymmetric system. For instance, in Fig. 6, $\rho_{1} \psi_{A 1}=0.75$ is illustrated by a vertical line that intersects the domain boundaries in $\mathrm{G}$ and $\mathrm{E}$, and curve $v a$ in $\mathrm{F}$, a two-layer system. The resulting segment GE is split into segments GF and FE, which describe layouts with different material location. In the upper region, there exist cross-sections coated with the stiffer material, whereas in the lower region, the stiffer material is slotted between the softer layers.

Applying a similar analysis for multiple asymmetric layers and merging the results with those presented in Section VI-B demonstrates that all the possible two-materials rectangular systems, including symmetric and asymmetric multilayers, fall within two boundaries describing three-symmetric-layers systems. No members of the Rectangles family can fall outside them.

In the following section, the maps are used to optimize the design of a bimaterial microresonator.

\section{DESIGN StUdY}

Recently, the materials world and the fabrication process for microdevices have seen a prolific expansion. The list of available materials is no longer limited to silicon. Polymers, ceramics, alloys, glasses, and composites have enlarged the spectrum of choice. Besides materials, the latest microfabrication technologies enable machine multimaterials systems.

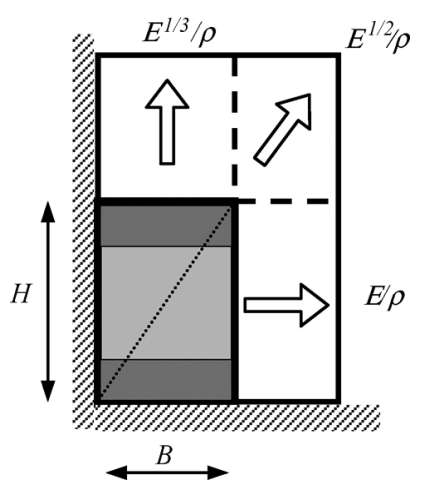

Fig. 7. Flexural response indexes for horizontal, proportional, and vertical scaling.

TABLE III

BULK MATERIAL PROPERTIES

\begin{tabular}{lll}
\hline \hline Material & $\mathrm{E}(\mathrm{GPa})$ & $\rho\left(\mathrm{Mg} \mathrm{m}^{-3}\right)$ \\
\hline $\mathrm{SiC}$ & 400 & 3.2 \\
$\mathrm{Si}$ & 160 & 2.3 \\
$\mathrm{Al}$ & 70 & 2.7 \\
$\mathrm{Ti}$ & 120 & 4.5 \\
$\mathrm{Cu}$ & 120 & 8.9 \\
\hline \hline
\end{tabular}

Compared to monolithic structures, such laminated composites have the potential of tripling the resonance frequency performance, as shown in Table II. In this design study, we explore the layers geometries that enable such a boost of performance for different bimaterial systems.

The design focus is on a rectangular cantilever resonator, which has prescribed stiffness, eigenmode, uniform length, and boundary conditions. The variables to contrasts are layer arrangement and materials. Different design constraints are applied to the size of the rectangular cross-section. These identify four scenarios. The first one, referred to as constrained envelope design, is the common scenario of composite design, which imposes restrictions on both dimensions of the cross-section. The other three scenarios are illustrated in Fig. 7. Here the envelope is left free to be scaled only in certain directions to meet the functional requirements. These conditions impose the candidates to be relatively scaled in horizontal, proportional, or vertical direction.

Among suitable materials for microresonating beams, we preselect two sets of bimaterial systems. The first one includes the metallization of silicon with aluminum, titanium, copper, or silicon carbide. The second set groups candidates where $\mathrm{SiC}$ is bonded to $\mathrm{Si}, \mathrm{Al}, \mathrm{Ti}$, or $\mathrm{Cu}$. Table III lists their material properties. The reason for selecting these two sets of structures is manifold. First, such materials are nowadays commonly used for microfabricated structures [50]-[57]. Secondly, their performance as monolithic structure has been already examined in works for material selection in micromechanical design and, thus, these results can be compared to those presented here [42], [47]. Lastly, such systems have been the focus of a recent study that had determined the frequency dependence of thermoelastic damping in three symmetric laminate beams [55]. These results show the detrimental effect of coating $\mathrm{Si}$ and $\mathrm{SiC}$, a choice that leads to an increase of damping as a function of the volume fraction. 


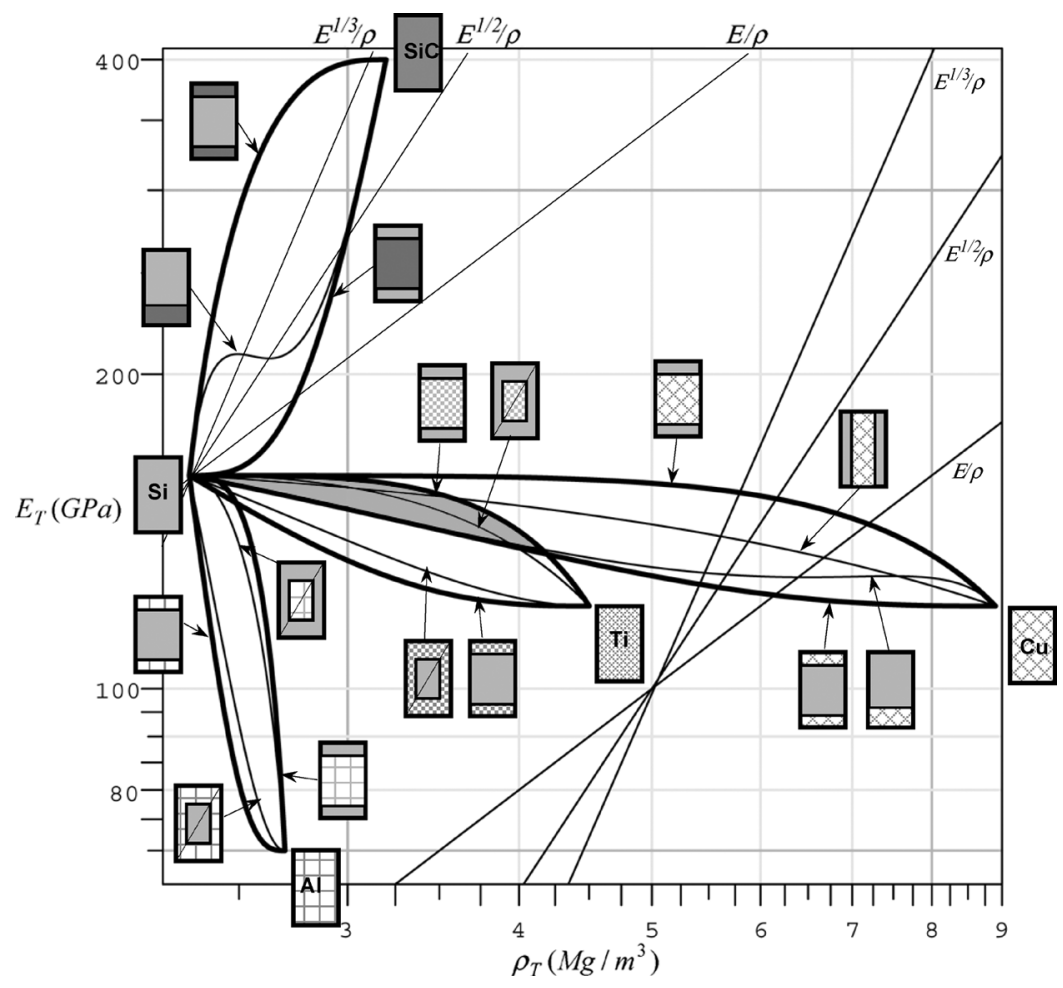

Fig. 8. Resonance frequency domains of $\mathrm{Si}$ combined, respectively, with $\mathrm{Al}, \mathrm{Ti}, \mathrm{Cu}, \mathrm{SiC}$.

To tackle the design, we do no solve the entire problem; rather we plot on properties maps the flexural response indexes, appropriate to the four design scenarios. The indexes are reported here.

- Constrained Envelope Design: When the cross-section sizes are constrained, the resonance frequency model is given by (10) and the optimization index for the rectangular resonator is $E_{T} / \rho_{T}$.

- Free Envelope Design: (Fig. 7) When cross-sections are relatively scaled to meet the design requirements, then $E_{T} / \rho_{T}$ is usually not appropriate. Indeed, for an arbitrary cross-section scaling, the general index is $E_{T}^{q} / \rho_{T}$, where $q=\ln u \nu / \ln u \nu^{3}$ is a scaling parameter [49]. Thus, this index, applied to the three scaling conditions of this case study (Fig. 7), becomes:

$E_{T} / \rho_{T}$ for horizontal scaling, where $u=1$;

$E_{T}^{1 / 2} / \rho_{T}$ for proportional scaling, $u=v$;

$E_{T}^{1 / 3} / \rho_{T}$ for a width constraint imposing vertical scaling, $v=1$.

Plotted in Figs. 8 and 9 on logarithmic scale axis, each index is identified by an isofrequency response line of the scaling condition. Each line guides the screening and the selection of the candidates for the four design scenarios. Points above a line have higher vibration frequencies than those below. As can been observed, changing the scaling condition may have a large impact into the ranking of candidates.

For a first screening, the boundaries of the Rectangles family can be plotted for each bimaterial system. These are illustrated as bold lines in Fig. 8 for $\mathrm{Si}$ bonded to $\mathrm{SiC}, \mathrm{Al}, \mathrm{Ti}, \mathrm{Cu}$ and in Fig. 9 for $\mathrm{SiC}$ combined with $\mathrm{Si}, \mathrm{Al}, \mathrm{Ti}$, and $\mathrm{Cu}$. The maps show that the monotonic increase, or decrease, of the domain bound- aries is governed by the relative ratios of the material properties. For instance, in Fig. 8, Si-SiC curves have positive derivative, whereas those describing the metallization of $\mathrm{Si}$ with the other metals have a negative gradient.

The boundaries of each bimaterial system describe three symmetric laminate composites for the whole range of volume fraction. Within them there exist all the possible two-materials systems containing two, three, and multiple layers arranged symmetrically and asymmetrically about the Envelope mid-plane. The upper boundary describes the coating of the stiffer material, as opposed to the lower limiting curves, which refer to the metallization of the stiff core by two softer material layers.

If the isoperformance lines with $q=1 / 3,1 / 2,1$ are set on silicon in Fig. 8 and on silicon carbide in Fig. 9, then the ranking of bimaterial candidates emerge for each of the scaling conditions. The following are the main observations that can be drawn when a monolithic structure is compared to single and two-materials systems.

1) Fig. 8 shows that $\mathrm{Si}$ provides a better frequency response than the monolithic $\mathrm{Al}, \mathrm{Ti}, \mathrm{Cu}$ structures in all four design scenarios. Between $\mathrm{SiC}$ and $\mathrm{Si}$, the former prevails for proportional, horizontal scaling, and fixed envelope design. This confirms recent results that have examined only the condition of fixed Envelope design, where the optimization index is $E_{T} / \rho_{T}$ [45]-[47]. However, if the candidates are constrained in width, then $E_{T}^{1 / 3} / \rho_{T}$ applies, and Si results in being better than $\mathrm{SiC}$.

2) Fig. 8 illustrates also that a monolithic $\mathrm{Si}$ resonator is still better than the bimaterial systems $\mathrm{Si}-\mathrm{Al}, \mathrm{Si}-\mathrm{Ti}$, and $\mathrm{Si}-\mathrm{Cu}$ for all the possible layers geometries in the four design scenarios. But this is not the case when $\mathrm{Si}$ is compared to 


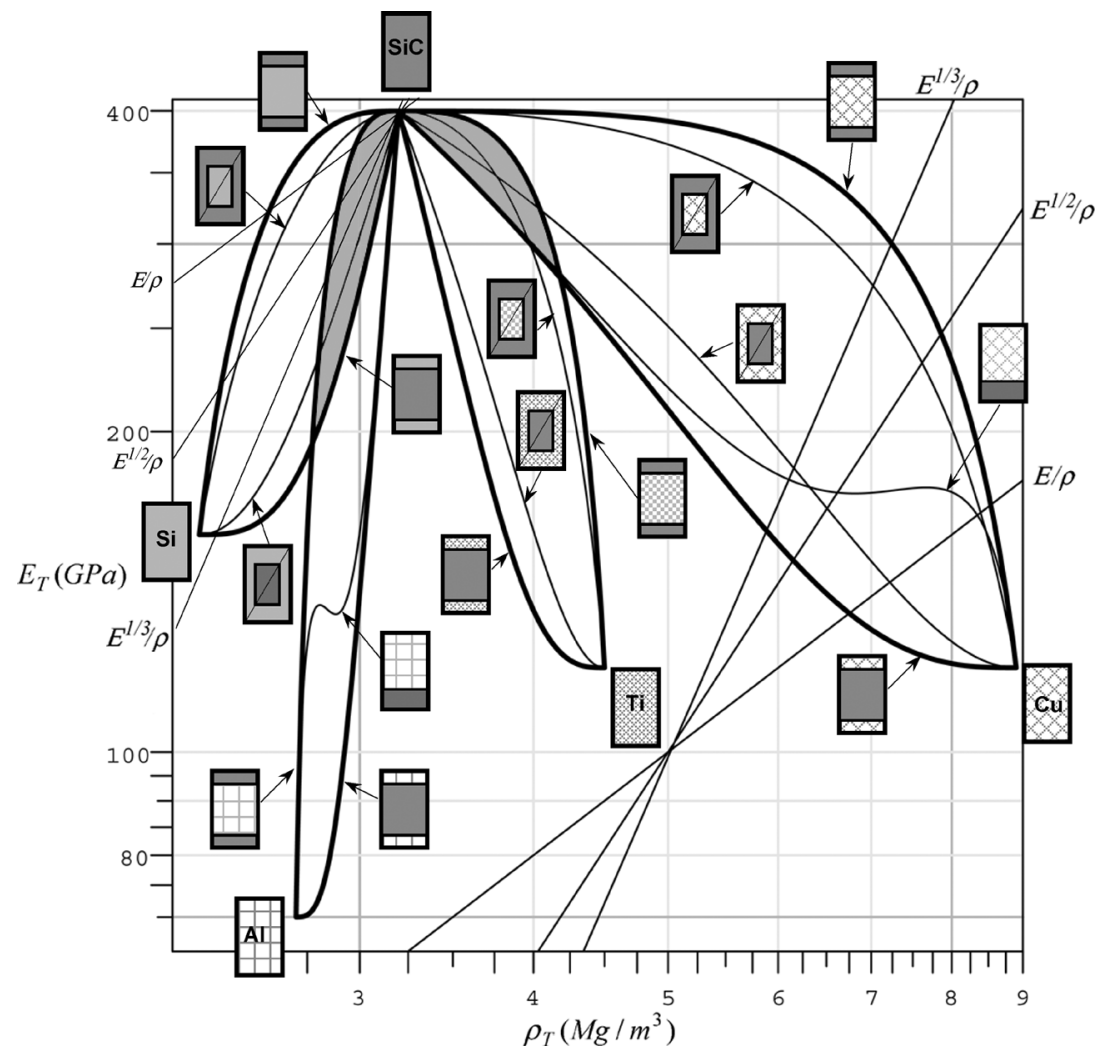

Fig. 9. Resonance frequency domains of $\mathrm{SiC}$ combined with $\mathrm{Al}, \mathrm{Ti}, \mathrm{Cu}, \mathrm{Si}$.

the $\mathrm{Si}$-SiC domain. The systems lying above the isoperformance lines show that combining $\mathrm{Si}$ with $\mathrm{SiC}$ in a certain volume fraction range provides higher flexural vibrations than $\mathrm{Si}$. The intersection of each line with the domain boundaries determines the volume fraction threshold for each scaling condition.

3) Figs. 8 and 9 show that an increase of the volume fraction of $\mathrm{SiC}$ in the upper boundary of the domain $\mathrm{Si}-\mathrm{SiC}$ maximizes flexural frequency. This curve is a cubic function of the dimensionless thickness, as reported in Table II. For the same system, a recent result has shown there is also a monotonic increase of thermoelastic damping with the volume fraction of $\mathrm{SiC}$ [55]. These two results could be combined to obtain the best volume fraction tradeoff that optimizes flexural vibrations and minimizes damping.

4) Fig. 9 confirms that a monolithic $\mathrm{SiC}$ is worse than a monolithic Si only for width constrained design. The maps, however, show that bonding $\mathrm{Al}$ with $\mathrm{SiC}$ may benefit the flexural response. Layouts falling into $\mathrm{SiC}-\mathrm{Al}$ domain above each isoperformance line have better vibration responses than the monolithic silicon carbide. This is in contrast to the effect of metalizing $\mathrm{Al}$ with $\mathrm{Si}$ in Fig. 8.

5) Competing bimaterial layered concepts have been shaded in Fig. 8 for Si-Ti and Si-Cu and in Fig. 9 for SiC-Si versus $\mathrm{SiC}-\mathrm{Al}$ and $\mathrm{SiC}-\mathrm{Ti}$ versus $\mathrm{SiC}-\mathrm{Cu}$. The gray regions identify the bilayer concepts that, although obtained with different materials and geometries, provide the same flexural responses.

After a first inspection, the screening may be refined to explore the impact of alternative layered geometries. As demon- strated in previous sections, no alternative layer concepts can lie outside a domain. Hence, all the expressions of the Rectangles classes (Table II) can be plotted within each domain. Only certain concepts, though, have been displayed in Figs. 8 and 9 to ensure readability.

Fig. 8 illustrates the limits of the proportionally scaled layers class for $\mathrm{Si}-\mathrm{Ti}$ and $\mathrm{Si}-\mathrm{Al}$ systems. For a given volume fraction, a full coating of Si can improve the flexural responses better than the case where $\mathrm{Si}$ is coated by $\mathrm{Ti}$ and $\mathrm{Al}$. For $\mathrm{Si}-\mathrm{Cu}$ domain, widthwise layer curves are visualized together with bilayered structures. The effect of asymmetry for two-layers systems has been plotted also for SiC-Si. In Fig. 9, on the other hand, fully coated concepts are visualized for $\mathrm{SiC}-\mathrm{Si}, \mathrm{SiC}-\mathrm{Cu}$, and $\mathrm{SiC}-\mathrm{Ti}$, while bilayered systems are shown for $\mathrm{SiC}-\mathrm{Al}$ and $\mathrm{Si}-\mathrm{Cu}$.

The above and other insights can be obtained by comparing different layered systems. It is noticed that although the focus of this paper has been only on the Rectangles family, other shape concepts, such as those listed in Table I, can be visualized and their performance compared for constrained and unconstrained design.

\section{CONCLUSION}

The flexural responses of a multilayered microresonator can be optimized by exploiting the geometry and the material potential. Cross-section shape, arrangement, symmetry, and number of layers, as well as volume fraction and material properties, govern the frequency response of a resonator with prescribed boundaries conditions, length and eigenmode. The impact of such factors has been visualized into efficiency maps, displaying the relation between flexural modulus and density. The charts 
demonstrate that although a bimaterial rectangular system can evolve in several symmetric and asymmetric multilayers, all the possible configurations are enclosed between two curves, describing three symmetric layers sandwiches. The maps ease the visual ranking, widen the spectrum of choices, and enable the contrast of bimaterial concepts with monolithic systems.

The method presented in this paper can be used to explore other performance metrics relevant to micromechanical design, such as strength and damping capacity. However, further work is required to demonstrate this. Limits due to fabrication techniques, and other detrimental issues, such as residual stresses among sacrificial layers, could also be integrated in the maps.

\section{ACKNOWLEDGMENT}

The author thanks Prof. S. Vengallatore of McGill University for a critical review of the manuscript.

\section{REFERENCES}

[1] C. W. de Silva, Vibration: Fundamental and Practice. Boca Raton, FL: CRC Press, 2000.

[2] S. D. Senturia, Microsystem Design. Norwell, MA: Kluwer, 2001.

[3] T. Kobayashi, J. Ohsawa, T. Hara, and N. Yamaguchi, "Contactless measurement of Young's modulus using laser beam excitation and detection of vibration of thin-film microresonators," J. Appl. Phys., vol. 43, pp. 1178-1182, 2004.

[4] J. W. M. Chon, P. Mulvaney, and J. E. Sader, "Experimental validation of theoretical models for the frequency response of atomic force microscope cantilever beams immersed in fluids," J. Appl. Phys., vol. 87, pp. 3978-3988, 2000.

[5] J. E. Sader, "Frequency response of cantilever beams immersed in viscous fluids with applications to the atomic force microscope," J. Appl. Phys., vol. 84, pp. 64-76, 1998.

[6] C. Serre, A. Perez-Rodriguez, J. R. Morante, P. Gorostiza, and J. Esteve, "Determination of micromechanical properties of thin films by beam bending measurements with an atomic force microscope," Sens. Actuators, vol. 74, pp. 134-138, 1999.

[7] P. A. Rasmussen, J. Thaysen, O. Hansen, S. C. Eriksen, and A. Boisen, "Optimised cantilever biosensor with piezoreistive read-out," Ultramicroscopy, vol. 97, no. 1, pp. 371-376, 2003.

[8] T. Roessig, A. P. Pisano, and R. T. Howe, "Surface-micromachined resonant force sensor," in Proc. ASME Int. Mech. Eng. Congr. Expo., 1995, pp. 871-876.

[9] Y. S. Hong, J. H. Lee, and S. H. Kim, "A laterally driven symmetric micro-resonator for gyroscopic applications," J. Micromech. Microeng., vol. 10, pp. 452-458, 2000.

[10] O. Degani, D. J. Seter, E. Socher, S. Kaldor, and Y. Nemirovsky, "Optimal design and noise consideration of micro machined vibrating rate gyroscope with modulated integrative differential optical sensing," $J$. Microelectromech. Syst., vol. 7, pp. 329-338, 1998.

[11] C. T.-C. Nguyen, L. P. B. Katehi, and G. M. Rebeiz, "Micromachined devices for wireless communications," Proc. IEEE, vol. 86, pp. 1756-1768, Aug. 1998.

[12] C. T.-C. Nguyen, "Vibrating RF MEMS for next generation wireless applications," in Proc. 2004 IEEE Custom Integr. Circuits Conf., Orlando, FL, Oct. 3-6, 2004, pp. 257-264.

[13] M. Sova and I. Bogdan, "Coplanar waveguide resonator design for array antenna applications," in Proc. 6th Int. Conf. Telecommun. Modern Satellite, Cable Broadcast. Service, 2003, vol. 1, pp. 57-59.

[14] C. T.-C. Nguyen, "Frequency-selective MEMS for miniaturized lowpower communication devices," IEEE Trans. Microwave Theory Tech., vol. 47, no. 8, pp. 1486-1503, 1999.

[15] A. C. Wong and C. T.-C. Nguyen, "Micromechanical mixer-filters," $J$. Microelectromech. Syst., vol. 13, pp. 100-112, 2004.

[16] C. T.-C. Nguyen, "Micromechanical resonators for oscillators and filters," in Proc. IEEE Ultrason. Symp., 1995, vol. 1, pp. 489-499.

[17] D. Galayko, A. Kaiser, B. Legrand, L. Buchaillot, D. Collard, and C. Combi, "Tunable bandpass T-filter with electrostatically-driven polysilicon micromechanical resonators," Sens. Actuators A, Phys., vol. 117, pp. 115-120, 2005.
[18] J. W. L. Zhou, H.-Y. Chan, T. K. H. To, K. W. C. Lai, and W. J. $\mathrm{Li}$, "Polymer MEMS Actuators for Underwater Micromanipulation," IEEE/ASME Trans. Mechatronics, vol. 9, no. 2, pp. 334-342, 2004.

[19] A. M. Taka, A. Omodaka, N. Takeshima, and H. Fujita, "Fabrication and operation of polyimide bimorph actuators for a ciliary motion system," J. Microelectromech. Syst., vol. 2, pp. 146-150, 1993.

[20] D. Lange, C. Hagleitner, C. Herzog, O. Brand, and H. Baltes, "Magnetic actuation and MOS-transistor sensing for CMOS-integrated resonators," in Proc. 15th IEEE Int. Conf. Microelectromech. Syst., 2002, pp. 304-307.

[21] J. Thaysen, A. D. Yalcinkaya, P. Vettiger, and A. Menon, "Polymerbased stress sensor with integrated readout," J. Phys. D, Appl. Phys., vol. 35, pp. 2698-2703, 2002.

[22] R. Melamud, B. Kim, M. A. Hopcroft, S. Chandorkar, M. Agarwal, C. Jha, S. Bhat, K. K. Park, and T. W. Kenny, "Composite flexural mode resonator with reduced temperature coefficient of frequency," in Proc. Solid-State Sens, Actuators, Microsyst. Workshop, Jun. 4-8, 2006.

[23] R. Marie, H. Jensenius, J. Thaysen, C. B. Christensen, and A. Boisen, "Adsorption kinetics and mechanical properties of thiol-modified DNA-oligos on gold investigated by microcantilever sensors," Ultramicroscopy, vol. 91, pp. 29-36, 2002.

[24] A.-R. A. Khaled, K. Vafai, M. Yang, X. Zhang, and C. S. Ozkan, "Analysis, control and augmentation of microcantilever deflections in biosensing systems," Sens. Actuators, vol. 94, pp. 103-115, 2003.

[25] M. Yue, H. Lin, D. E. Dedrick, S. Satyanarayana, A. Majumdar, A. Bedekar, S. J. W. Jenkins, and S. Sundaram, "A 2-D microcantilever array for multiplexed biomolecular analysis," J. Microelectromech. Syst., vol. 13, pp. 290-299, 2004.

[26] K. Wang, A.-C. Wong, and C. T.-C. Nguyen, "VHF free-free beam high-Q micromechanical resonators," J. Microelectromech. Syst., vol. 9, no. 3, pp. 347-360, 2000.

[27] S. Lin, "Effect of electric load impedances on the performance of sandwich piezoelectric transducers," IEEE Trans. Ultrason., Ferroelectr., Freq. Control, vol. 51, no. 10, 2004.

[28] _ , "Piezoelectric ceramic rectangular transducers in flexural vibration," IEEE Trans. Ultrason., Ferroelectr., Freq. Control, vol. 51, no. 7, 2004

[29] A. T. Ferguson, L. Li, V. T. Nagaraj, B. Balachandran, B. Piekarski, and D. L. DeVoe, "Modeling and design of composite free-free beam piezoelectric resonators," Sens. Actuators, vol. 118, no. 1, pp. 63-69, 2005.

[30] R. Sandberg, A. Boisen, and W. Svendsen, "Characterization system for resonant micro- and nanocantilevers," Rev. Sci. Instrum., 2005.

[31] R. Sandberg, K. Mølhave, A. Boisen, and W, Svendsen, "Effect of gold coating on the Q-factor of a resonant cantilever," J. Micromech. Microeng, vol. 15, pp. 2249-2253, 2005.

[32] — - "Temperature and pressure dependence of resonance in multi-layer microcantilevers," J. Micromech. Microeng., vol. 15, pp. 1454-1458, 2005.

[33] J. Mertens, E. I. Finot, T. Thundat, A. Fabre, M.-H. Nadal, V. Eyraud, and E. Bourillot, "Effects of temperature and pressure on microcantilever resonance response," Ultramicroscopy, vol. 97, no. 1, pp. 119-126, 2003.

[34] H. L. Cox, The Design of Structures of Least Weight. Oxford, U.K. Pergamon, 1965.

[35] F. R. Shanley, Weight-Strength Analysis of Aircraft Structures, 2nd ed. New York: Dover, 1960.

[36] J. B. Caldwell and R. G. Woodhead, "Ship structures: some possibilities for improvement," Trans. North East Coast Inst. Eng. Shipbuild., vol. 89, pp. 101-120, 1973.

[37] J. G. Parkhouse, "Structuring a process of material dilution," in Proc. 3rd Int. Conf. Space Struct., 1984, pp. 367-374.

[38] M. F. Ashby, "Materials and shape," Acta Metall. Mater., vol. 39, no. 6, pp. 1025-1039, 1991

[39] - "Criteria for selecting the components of composites," Acto Metall. Mater., vol. 41, no. 5, pp. 131-135, 1993.

[40] —-, Material Selection in Mechanical Design. Oxford, U.K.: Pergamon, 1999.

[41] M. F. Ashby and Y. J. M. Bréchet, "Designing hybrid materials," Acta Materialia, vol. 51, pp. 5801-5821, 2003.

[42] D. Cebon and M. F. Ashby, "Materials selection for precision instruments," Meas. Sci. Technol., vol. 5, pp. 296-306, 1994.

[43] J. E. Huber, N. A. Fleck, and M. F. Ashby, "The selection of mechanical actuators based on performance indices," in Proc. Roy. Soc. London A, 1997, vol. 453, pp. 2185-2205.

[44] J. Shieh, J. E. Huber, N. A. Fleck, and M. F. Ashby, "The selection of sensors," Progr. Mater. Sci., vol. 46, pp. 461-504, 2001. 
[45] S. M. Spearing, "Materials issues in microelectromechanical systems (MEMS)," Acta Mater., vol. 48, pp. 179-196, 2000.

[46] V. T. Srikar and S. M. Spearing, "Materials selection for microfabricated electrostatic actuators," Sens. Actuators, vol. 102A, pp. 279-285, 2003.

[47] - "Materials selection in micromechanical design: An application of the Ashby approach," J. Microelectromech. Syst., vol. 12, pp. 3-10, 2003.

[48] D. Pasini, D. J. Smith, and S. C. Burgess, "Structural efficiency maps for beams subjected to bending," Proc. Inst. Mech. Eng., Part L: J. Mater. Design Applicat., vol. 217, no. 3, pp. 207-220, 2003.

[49] D. Pasini, "Shape transformers for material and shape selection of lightweight beams," J. Mater. Design, 2006, to be published.

[50] K. I. Jolic, M. K. Ghantasala, J. P. Hayes, and H. Jin, "Fabrication of three-dimensional inductor coil using excimer laser micromachining," J. Micromech. Microeng., vol. 13, no. 5, pp. 782-789, 2003.

[51] C. R. Stoldt, C. Carraro, W. R. Ashurst, D. Gao, R. T. Howe, and R. A. Maboudian, "Low-temperature CVD process for silicon carbide MEMS," Sens. Actuators A, vol. 97-98, pp. 410-415, 2002.

[52] S. Evoy, A. Olkhovets, L. Sekaric, J. M. Parpia, H. G. Craighead, and D. W. Carr, "Temperature-dependent internal friction in silicon nanoelectromechanical systems," Appl. Phys. Lett., vol. 77, pp. 2397-2399, 2000.

[53] X. M. H. Huang, K. L. Ekinci, Y. T. Yang, C. A. Zorman, M. Mehregany, and M. L. Roukes, "Nanoelectromechanical silicon carbide resonators for ultra-high frequency applications," in Proc. 2002 Sensor, Actuator Microsyst. Workshop, Hilton Head, SC, Jun. 2-6, 2002, pp. 368-369.

[54] R. F. Wiser, M. Tabib-Azar, M. Mehregany, and C. A. Zorman, "Polycrystalline silicon-carbide surface-micromachined vertical resonatorspart II: electrical testing and material property extraction," J. Microelectromech. Syst., vol. 14, no. 3, pp. 579-589, Jun. 2005.
[55] S. Vengallatore, "Analysis of thermoelastic damping in laminated composite micromechanical beam resonators," J. Micromech. Microeng., vol. 15, pp. 2398-2404, 2005.

[56] L. Li, P. Kumar, S. Kanakraju, and D. L. DeVoe, "Piezoelectric AlGaAs bimorph microactuators," J. Micromech. Microeng., vol. 16, pp. 1062-1066, 2006.

[57] N. Cheggour, J. W. Ekin, C. L. H. Thieme, Y.-Y. Xie, V. Selvamanickam, and R. Feenstra, "Reversible axial-strain effect in Y-Ba-Cu-O coated conductors," Supercond. Sci. Technol., vol. 18, no. 12, pp. S319-S324, 2005.

Damiano Pasini (M'04) received the Master's degrees in civil engineering and in architectural engineering in 1995 and 2000, respectively, from the University of Pavia, Italy. In 2003, he received the Ph.D. degree in mechanical engineering from the University of Bristol, U.K., with a dissertation on the optimization of material, shape, and structural form.

From 1995 to 2000, he worked in Italy as a Professional Civil Engineer. In 2003, he joined the German Aerospace Center (D.L.R.) in the Department of Adaptronics at the Institute of Structural Mechanics, Braunschweig, Germany. Here, his research focused on the optimization of solid-state hinges for adaptive wing flaps. Since July 2004, he has been Assistant Professor in the Department of Mechanical Engineering of McGill University. His main research interests hinge on the optimum design of engineering structures and biomaterials at different length scale.

Dr. Pasini is a Member of the McGill Institute for Advanced Materials and the American Society of Mechanical Engineers (ASME). 